\title{
Donor-Matched Comparison of Chondrogenic Potential of Equine Bone Marrow- and Synovial Fluid- Derived Mesenchymal Stem Cells: Implications for Cartilage Tissue Regeneration
}

\author{
Mohammed Zayed ${ }^{1,2}$, Christopher Caniglia ${ }^{3}$, Nabil Misk $^{4}$ and Madhu S. Dhar ${ }^{1 *}$ \\ 'Department of Large Animal Clinical Sciences, College of Veterinary Medicine, University of Tennessee, Knoxville, TN, USA, \\ ${ }^{2}$ Department of Animal Surgery, College of Veterinary Medicine, South Valley University, Qena, Egypt, ${ }^{3}$ Manor Equine \\ Hospital, Monkton, MD, USA, ${ }^{4}$ Department of Animal Surgery, College of Veterinary Medicine, Assiut University, Asyut, Egypt
}

OPEN ACCESS

Edited by: Debbie Guest,

Animal Health Trust, UK

Reviewed by:

Rebeca Blázquez Durán, Centro de Cirugía de Mínima Invasión Jesús Usón, Spain Helen Elizabeth McCarthy,

Cardiff University, UK

${ }^{*}$ Correspondence: Madhu S. Dhar mdhar@utk.edu

Specialty section: This article was submitted to Veterinary Regenerative Medicine,

a section of the journal

Frontiers in Veterinary Science

Received: 12 September 2016 Accepted: 19 December 2016

Published: 18 January 2017

Citation:

Zayed M, Caniglia C, Misk N and Dhar MS (2017) Donor-Matched

Comparison of Chondrogenic Potential of Equine Bone Marrowand Synovial Fluid-Derived Mesenchymal Stem Cells: Implications for Cartilage Tissue Regeneration.

Front. Vet. Sci. 3:121. doi: 10.3389/fvets.2016.00121
Mesenchymal stem cells (MSCs) have been demonstrated to be useful for cartilage tissue regeneration. Bone marrow (BM) and synovial fluid (SF) are promising sources for MSCs to be used in cartilage regeneration. In order to improve the clinical outcomes, it is recommended that prior to clinical use, the cellular properties and, specifically, their chondrogenic potential must be investigated. The purpose of this study is to compare and better understand the in vitro chondrogenic potential of equine bone marrow-derived mesenchymal stem cells (BMMSCs) and synovial fluid-derived mesenchymal stem cells (SFMSCs) populated from the same equine donor. BM- and SF-derived MSCs cultures were generated from five equine donors, and the MSCs were evaluated in vitro for their morphology, proliferation, trilineage differentiation, and immunophenotyping. Differences in their chondrogenic potentials were further evaluated quantitatively using glycosaminoglycan (GAG) content and via immunofluorescence of chondrogenic differentiation protein markers, SRY-type HMG box9, Aggrecan, and collagen II. The BMMSCs and SFMSCs were similar in cellular morphology, viability, and immunophenotype, but, varied in their chondrogenic potential, and expression of the key chondrogenic proteins. The SFMSCs exhibited a significant increase in GAG content compared to the BMMSCs $(P<0.0001)$ in three donors, suggesting increased levels of chondrogenesis. The expression of the key chondrogenic proteins correlated positively with the GAG content, suggesting that the differentiation process is dependent on the expression of the target proteins in these three donors. Our findings suggest that even though SFMSCs were hypothesized to be more chondrogenic relative to BMMSCs, there was considerable donor-to-donor variation in the primary cultures of MSCs which can significantly affect their downstream application.

Keywords: chondrogenesis, mesenchymal stem cells, bone marrow, synovial fluid, cartilage regeneration, horse

Abbreviations: MSCs, mesenchymal stem cells; BM, bone marrow; SF, synovial fluid; BMMSCs, bone marrow-derived mesenchymal stem cells; SFMSCs, synovial fluid-derived mesenchymal stem cells; GAG, glycosaminoglycan; SOX9, SRY-type HMG box9; Col II, collagen type II. 


\section{INTRODUCTION}

Osteoarthritis (OA) is a chronic disease pertaining to progressive deterioration of the articular cartilage and subchondral bone (1). $\mathrm{OA}$ is a major cause of reduced performance and loss in human and equine population (2-4). Attempts have been made to treat $\mathrm{OA}$ and prevent joint degeneration, which primarily includes injection of pain relief medications to reduce inflammation and treat pain (5). Since, cartilage degeneration is a sequel to OA, aggressive surgical approaches such as chondrocyte implantation have also been used in the treatment of massive chondral injuries in human and equine patients $(6,7)$. To date, no therapies are available to effectively regenerate the affected tissue, posing $\mathrm{OA}$ as a major health concern. In recent years, mesenchymal stem cells (MSCs) have shown promise in human and equine regenerative medicine and have been used in tissue regeneration and treatment of many diseases including those affecting the joint (8-12).

Equine adult MSCs are multipotent progenitor cells that can be isolated from various adult tissues, including bone marrow $(\mathrm{BM})$, adipose tissue (AD), and peripheral blood (13-16). Recently, MSCs generated from equine synovial fluid (SF) have been shown to be multipotent in vitro $(17,18)$. Even though MSCs can be easily isolated from various sources, researchers have reported differences regarding their basic biological properties, in vitro. These differences include proliferation, differentiation, and immunomodulatory effect, which can adversely impact their function and hence, the in vivo application (19-21). Furthermore, variations or differences can occur in the properties of MSCs generated not only from different sources but also from the same source in different donors. For instance, we reported a study where we observed differences within bone marrow-derived mesenchymal stem cells (BMMSCs) in a group of age- and gender-matched horses (22).

Bone marrow is an acceptable adult stem cell source used in cartilage tissue engineering $(23,24)$. However, possible contamination during BM aspiration, decrease in proliferation and chondrogenic potential with age, hypertrophy, and mineralization when implanted in vivo hinder its clinical application (25). Hence, BMMSCs may not be the best source for cartilage repair. Recently, it has been recognized in both human and equine medicine that SF collection is relatively easy, less invasive, and a rich source of MSCs. In addition, the synovial MSCs have greater proliferative and chondrogenic abilities than other MSCs, making SF or the synovium cells as an alternative, accessible source of MSCs and a suitable candidate for cartilage repair $(17,26,27)$.

Although BM and SF represent attractive tissue sources for MSCs, there has been no comparison of the chondrogenic potential of these cells from the same donor. Based on published papers and the results from our laboratory, we hypothesize that equine synovial fluid-derived mesenchymal stem cells (SFMSCs) can be isolated and characterized in vitro and their chondrocyte differentiation would be superior to BMMSCs, but there may be donor-to-donor variations when donor-matched comparison is done. In order to prove our hypothesis, we performed a donormatched comparison of the biological properties of equine MSCs isolated from BM and SF. Differences, if any, in their chondrogenic potentials were evaluated using glycosaminoglycan (GAG) assay and by assessing changes in the key proteins of chondrogenic differentiation using immunofluorescence (IF).

\section{MATERIALS AND METHODS}

\section{Tissue Harvest and Isolation of MSCs}

All the experiments were conducted in accordance with the protocols approved by University of Tennessee Institutional Animal Care and Use Committee. Five healthy, mixed breed horses, aged 8-13 years were used for BM and SF collections.

\section{Bone Marrow}

All procedures were performed as described previously $(22,28)$. Mononuclear fraction of cells from BM $(n=5)$ collected in the presence of $100 \mathrm{IU} / \mathrm{ml}$ heparin (Celsus, Cincinnati, OH, USA) were seeded in the basal medium consisting of Dulbecco's Modified Eagle Medium/Ham's F-12 (Fisher Scientific, Pittsburgh, PA, USA), $10 \%$ fetal bovine serum (Merck Animal Health, Summit, NJ, USA), and $1 \%$ penicillin/streptomycin solution (Fisher Scientific, Pittsburgh, PA, USA) and incubated at $37^{\circ} \mathrm{C}$, with $5 \%$ $\mathrm{CO}_{2}$ (Fisher Scientific, Pittsburgh, PA, USA). Adherent cells were allowed to reach $70-80 \%$ confluency before being harvested with $0.25 \%$ trypsin EDTA (Fisher Scientific, Pittsburgh, PA, USA) for $2 \mathrm{~min}$ at $37^{\circ} \mathrm{C}$.

\section{Synovial Fluid}

Synovial fluid $(n=5)$ was obtained using methods described previously $(17,18)$ with slight modifications. Horses were sedated with detomidine $\mathrm{HCl}$ (Pfizer Animal Health, NY, USA) $(0.015-0.26 \mathrm{mg} / \mathrm{kg}$ bwt i.v.), $2-5 \mathrm{ml}$ of SF was collected aseptically from each of the radiocarpal, intercarpal, and tarsocrural joints. Approximately $0.5 \mathrm{ml}$ of each sample was collected in microtainer tubes containing EDTA (Fisher Scientific, Pittsburgh, PA, USA) and was analyzed for cytology. For proliferation and expansion of SFMSCs from confirmed clinically normal SF, $1 \mathrm{ml}$ of SF was diluted (1:10) with DMEM F-12 (Fisher Scientific, Pittsburgh, PA, USA) basal medium and seeded onto $75 \mathrm{~cm}^{2}$ vented tissue culture flasks (Fisher Scientific, Pittsburgh, PA, USA) and incubated at $37^{\circ} \mathrm{C}$, with $5 \% \mathrm{CO}_{2}$ (Fisher Scientific, Pittsburgh, PA, USA) for 4 days before the first medium change. Thereafter, medium was changed every $2-3$ days until the cells reached $70-80 \%$ confluency. Adherent cells were harvested with $0.25 \%$ trypsin EDTA (Fisher Scientific, Pittsburgh, PA, USA) for $1 \mathrm{~min}$ at $37^{\circ} \mathrm{C}$.

Each horse was sampled twice between 3 and 6 months. All the cell culture experiments were carried out in triplicate, and each assay was repeated with the equine MSCs from two independent BM and SF harvests.

\section{Nuclear/Cytoplasmic Staining}

Nuclear/cytoplasmic fluorescent staining was used to demonstrate the morphology of MSCs for cytoplasmic staining; MSCs were stained with $5 \mu \mathrm{g}$ of WGA (Life Technologies, Grand Island, NY, USA) (wheat germ agglutinin, Alexa Fluor 488 conjugate) for $10 \mathrm{~min}$. To stain the nucleus, cells were further washed and stained with $5 \mu \mathrm{g}$ of TO-PRO-3 iodide stain (Life Technologies, Grand Island, NY, USA) for $10 \mathrm{~min}$. After washing, the cells were mounted with Slow Fade Gold Antifade Reagent 
(Life Technologies, Grand Island, NY, USA), and images were obtained using a laser scanning spectral confocal microscope (Leica Microsystems@, Wetzlar, Germany).

\section{Cell Viability and Proliferation}

The cellular viability and proliferation of $2.0 \times 10^{4}$ cells of P2-P3 of expanded equine BMMSCs and SFMSCs from each donor $(n=5)$ was assessed at 2, 4, and 8 days using the CellTiter 96 Aqueous Non-Radioactive (MTS) assay (Promega, Madison, WI, USA), as described previously $(20,22)$. The optical density of the cell and the MTS reagent complex was measured on a microplate fluorescence reader (BioTek, Winooski, VT, USA) at $490 \mathrm{~nm}$. Medium without cells was used as a blank. Graph of absorbance at $490 \mathrm{~nm}$ vs. days of proliferation was generated.

\section{Immunophenotyping}

Flow cytometric analysis was performed in P2-P3 expanded BMMSCs and SFMSCs from each donor $(n=5)$ using a panel of cluster-of-differentiation (CD) antigen antibodies. Briefly, for each staining, $1 \times 10^{6}$ cells were harvested and collected in $1 \times$ florescence-activated cell sorting (FACS) buffer (phosphate-buffered saline with $0.1 \%$ bovine serum albumin) (Fisher Scientific, Pittsburgh, PA, USA). The Fc receptors were blocked by preincubating cells with $0.5 \mathrm{mg} / \mathrm{ml}$ mouse (BD Bioscience, Grand Island, NY, USA) Fc block for 20 min on ice. After repeated washing, cells were incubated with fluorescein isothiocyanate (FITC) preconjugated CD90 (BD Bioscience, Grand Island, NY, USA), CD29 (Beckman Coulter, Brea, CA, USA), CD34 (Millipore, Billerica, MA, USA), major histocompatibility complex II (MHC-II) (AbD Serotec, Raleigh, NC, USA), and non-conjugated CD44 (Abcam, Cambridge, MI, USA) primary antibodies for $60 \mathrm{~min}$ in the dark on ice. To detect CD44, cells were stained with the secondary antibody for $30 \mathrm{~min}$ on ice (Table 1). Negative control staining was performed using a FITC conjugated mouse IgG1 (BD Bioscience, Grand Island, NY, USA) isotype. The raw data of fluorescence were measured using the BD FACS Diva software (BD Bioscience, Grand Island, NY, USA). The percentages of cells positive for a given protein were recorded.

\section{Adipogenesis and Osteogenesis}

Roughly, $2.0 \times 10^{5}$ BMMSCs and SFMSCs from each donor $(n=5)$ were induced to adipogenesis and osteogenesis. Cells

TABLE 1 | Antibodies used for analyzing the specific molecular markers on the cell surface.

\begin{tabular}{llll}
\hline Protein marker & Clone & Dilution & Form \\
\hline CD90 & OX-7 & $1: 10$ & $\begin{array}{l}\text { Fluorescein } \\
\text { isothiocyanate (FITC) } \\
\end{array}$ \\
& & & $\begin{array}{l}\text { conjugate } \\
\text { FITC conjugate }\end{array}$ \\
CD34 & 581 & $1: 5$ & FITC conjugate \\
CD29 & 4B4LDC9LDH8 & $1: 5$ & Non-Conjugate \\
CD44 & IM7 & $1: 100$ & Nol \\
Major histocompatibility & CVS20 & $1: 10$ & FITC conjugate \\
complex II monomorphic & & & \\
Secondary (FITC) & - & $1: 100$ & Alexa Fluor 488 anti-rat \\
Isotype control (IgG1) & A85-1 & $1: 10$ & FITC conjugate
\end{tabular}

were seeded in tissue culture dishes in the basal medium, with the medium change every $2-3$ days. At $70-80 \%$ confluency, cells were induced to differentiate into each of the two lineages using lineage-specific differentiation media. Adipogenic differentiation was induced in complete growth medium supplemented with $1 \mu \mathrm{mol} / \mathrm{l}$ dexamethasone (BioTek, Winooski, VT, USA), $10 \mu \mathrm{g} /$ $\mathrm{ml}$ recombinant human insulin (BioTek, Winooski, VT, USA), $0.5 \mathrm{mmol} / \mathrm{l}$ 3-isobutyl-1-methylxanthine (BioTek, Winooski, VT, USA), 15\% rabbit serum (BioTek, Winooski, VT, USA), and $20 \mu \mathrm{mol} / \mathrm{l}$ indomethacin (BioTek, Winooski, VT, USA). Osteogenic differentiation was induced in complete growth medium supplemented with $100 \mathrm{nmol} / \mathrm{l}$ dexamethasone, $10 \mathrm{mmol} / \mathrm{l} \beta$-glycerophosphate (BioTek, Winooski, VT, USA), and $0.25 \mathrm{mmol} / \mathrm{l}$ ascorbic acid (BioTek, Winooski, VT, USA). Adipogenic and osteogenic differentiation was confirmed at day 7 and 14 using $0.5 \%$ Oil Red $\mathrm{O}$ staining and 2\% Alizarin Red S staining (Fisher Scientific, Pittsburgh, PA, USA), respectively. Stained cells were visualized and photographed using Nikon DS-Fi2 connected to a Zeiss microscope and evaluated using the NIS Elements imaging software (BioTek, Winooski, VT, USA).

\section{Chondrogenesis and GAG Content}

A total of $70-80 \%$ confluent $2.0 \times 10^{5}$ BMMSCs and SFMSCs from each donor $(n=5)$ were induced to chondrogenic differentiation. Differentiation was induced using complete growth medium supplemented with $100 \mathrm{nmol} / \mathrm{l}$ dexamethasone, $0.25 \mathrm{mmol} / \mathrm{l}$ ascorbic acid (Sigma-Aldrich, Saint Louis, MI, USA), and $5 \mathrm{ng} / \mathrm{ml}$ transforming growth factor beta (R\&D Systems, Minneapolis, MI, USA) for 14 days. To confirm chondrogenic differentiation, cells were stained with Alcian Blue $(\mathrm{pH}=2.5)$ (Fisher Scientific, Pittsburgh, PA, USA). To measure the GAG content, subsequent to staining, Alcian Blue dye was extracted by exposing the cells to $500 \mu \mathrm{l}$ of $6 \mathrm{M}$ guanidine/ $\mathrm{HCl}$ (Fisher Scientific, Pittsburgh, PA, USA) overnight with shaking at room temperature. The optical density of the extracted Alcian Blue was measured on a microplate fluorescence reader (BioTek, Winooski, VT, USA) at $620 \mathrm{~nm}$.

\section{IF Staining}

Bone marrow-derived mesenchymal stem cells and SFMSCs from each donor $(n=5)$ were cultured as described above until $70-80 \%$ confluent and were subsequently induced to differentiate into chondrocytes using the cocktail described above for 14 days. Differentiated cells were fixed with $4 \%$ paraformaldehyde for 10 min, permeabilized with $0.1 \%$ Triton X-100 (Sigma-Aldrich, Saint Louis, MI, USA) for $10 \mathrm{~min}$, and finally blocked with $1 \%$ Power Block (BioGenex, San Ramon, CA, USA) for $30 \mathrm{~min}$. For each analysis, SRY-type HMG box9 (SOX9) (Santa Cruz Biotechnology, Dallas, TX, USA), Aggrecan (Fisher Scientific, Pittsburgh, PA, USA), and collagen type II (Col II) (Abcam, Cambridge, MI, USA) were used to detect the target proteins (Table 2). Antigen detection was performed using specific secondary antibodies. Samples were mounted in Prolong Gold antifade reagent with DAPI (Life Technologies, Grand Island, NY, USA), and images were obtained with a laser scanning spectral confocal microscope (Leica Microsystems@ $@$, Wetzlar, Germany). Fluorescent-labeled secondary antibodies alone and FITClabeled anti-mouse immunoglobulin G1 were used as negative 
TABLE 2 | Differentiation time, dilution, and secondary antibodies for chondrogenic protein markers using immunofluorescence analysis.

\begin{tabular}{|c|c|c|c|c|}
\hline Protein marker & Differentiation day & Dilution & Concentration $(\mu \mathrm{g} / \mathrm{ml})$ & Secondary antibody \\
\hline SRY-type HMG box9 & 3 & $1: 100$ & 2 & Alexa fluor 647 anti-rabbit \\
\hline Aggrecan & 14 & $1: 200$ & 4 & Alexa Fluor 488 anti-mouse \\
\hline Collagen type II & 14 & $1: 200$ & 4 & Alexa Fluor 488 anti-rabbit \\
\hline
\end{tabular}

controls. Mean intensity fluorescence was quantified using the Leica TCS SP2 software by selecting at least four representative areas of the same settings.

\section{Statistical Analysis}

For the statistical analysis, all quantitative group data are shown as the mean $\pm \mathrm{SD}$. Data for cell proliferation, GAG content, immunophenotyping, and IF from the two cell sources were analyzed and compared using Student's $t$-test (SPSS version 23, SPSS, Chicago, IL, USA). Differences of $P<0.05$ were considered to be statistically significant.

\section{RESULTS}

\section{Isolation and In Vitro Expansion of MSCs}

Bone marrow-derived mesenchymal stem cells adhered to the tissue culture polystyrene-treated plastic within $48 \mathrm{~h}$ from seeding, proliferated, and maintained spindle-like morphology throughout the expansion process. The SFMSCs were rounded when seeded in culture, acquired spindle-like morphology within 4 days after seeding, proliferated, and maintained spindle-like morphology throughout the expansion process. The cellular morphology was monitored and observed up to passage 6 for both cell types. The morphology was confirmed using a standard nuclear/cytoplasmic fluorescent staining (Figures 1A,B).

\section{Cell Proliferation and Viability}

Cellular proliferation and viability of BMMSCs and SFMSCs were assessed through a period of 8 days using MTS assay (Figure 2). In presence of the growth medium provided, both cell types were viable and the cell numbers increased linearly over time, suggesting that both types of MSCs can be expanded in vitro to generate sufficient numbers for implantation.

\section{Immunophenotyping}

Flow cytometric analyses of BMMSCs and SFMSCs from all donors, revealed $>70 \%$ expression of MSCs markers including CD29, CD44, and CD90. The BMMSCs were negative for CD34, a hematopoietic marker, and MHC-II. Comparatively, the SFMSCs exhibited low levels of CD34 and significantly high expression of MHC-II. The average percentage of each marker with SD is illustrated in Table 3.

\section{Adipogenesis and Osteogenesis}

Subjective evaluation of the patterns of differentiation demonstrated that both BMMSCs and SFMSCs undergo differentiation in presence of the lineage-specific induction media. The adipogenic and osteogenic differentiation was demonstrated by the

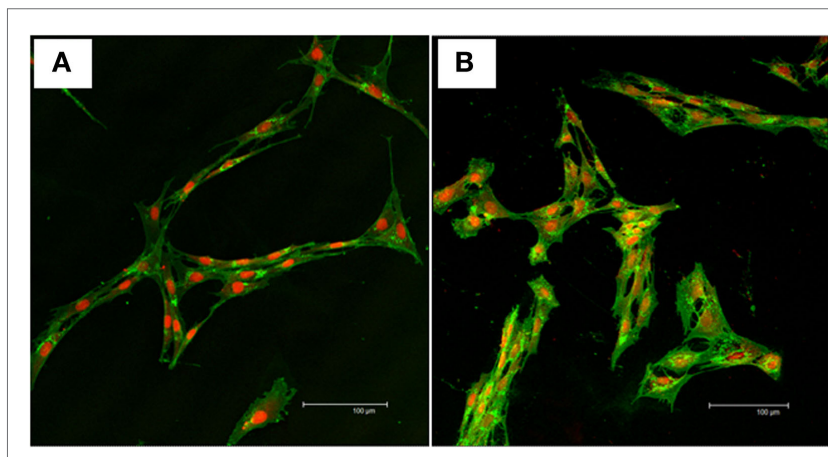

FIGURE 1 | Nuclear/cytoplasmic staining of mesenchymal stem cells. Representative confocal images of WGA (green) and TOPRO-3-iodide (red) fluorescent stains showing the viability and morphology of the cells.

(A) Typical fibroblast-like morphology of undifferentiated bone marrowderived mesenchymal stem cells. (B) Typical fibroblast-like morphology of undifferentiated synovial fluid-derived mesenchymal stem cells. Scale bar $=100 \mu \mathrm{m}$

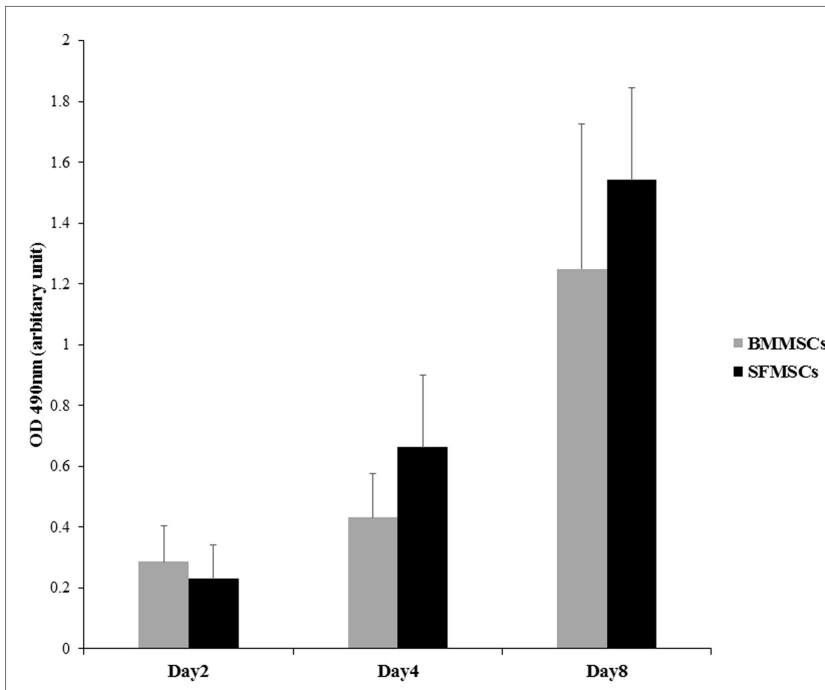

FIGURE 2 | Measurement of cell proliferation of bone marrow-derived mesenchymal stem cells (BMMSCs) and synovial fluid-derived mesenchymal stem cells (SFMSCs). MTS assay was used to evaluate the rate of proliferation over a period of 8 days. Absorbance, optical density at $490 \mathrm{~nm}$ is linearly related to the cell numbers and hence represents the proliferation at a given time point. Increase in the optical density between two time points can be used as an index to measure the rate of proliferation. Data are presented as means $\pm \mathrm{SD}$. Error bars represent the SD.

deposition of lipid droplets into the cytoplasm, as visualized by Oil Red $\mathrm{O}$ staining and calcium matrix precipitation using alizarin red dye, respectively (Figure 3). 
TABLE 3 | Percentage means of mesenchymal stem cells (MSCs) from bone marrow (BM) and synovial fluid (SF) of the same donor for the CD90, CD29, CD44, CD34, and major histocompatibility complex II (MHC-II) markers by flow cytometry.

\begin{tabular}{|c|c|c|c|c|c|}
\hline Donors & CD90 & CD29 & CD44 & CD34 & MHC-II \\
\hline Bone marrow-derived mesenchymal stem cells (BMMSCs) donor 1 & $95.55 \pm 0.55$ & $75.6 \pm 3$ & $96.6 \pm 0$ & $1.15 \pm 0.85$ & $0.28 \pm 0$ \\
\hline Synovial fluid-derived mesenchymal stem cells (SFMSCs) donor 1 & $83.35 \pm 2.35$ & $90.55 \pm 1.65$ & $91.5 \pm 4.5$ & $0.95 \pm 0.75$ & $3.245 \pm 2.65$ \\
\hline BMMSCs donor 2 & $90.95 \pm 4.35$ & $65.95 \pm 7.3$ & $99.1 \pm 0$ & $1.05 \pm 0.05$ & $1.36 \pm 0.36$ \\
\hline SFMSCs donor 2 & $76.3 \pm 6$ & $71.55 \pm 6.75$ & $94.25 \pm 1.25$ & $1.45 \pm 0.35$ & $7.805 \pm 5.1$ \\
\hline BMMSCs donor 3 & $97.25 \pm 2.55$ & $86.35 \pm 12.15$ & $92.65 \pm 7.3$ & $0.635 \pm 0.36$ & $1.85 \pm 0.45$ \\
\hline SFMSCs donor 3 & $98.9 \pm 0.5$ & $99.75 \pm 0.25$ & $99.9 \pm 0$ & $10.25 \pm 0.25$ & $10.2 \pm 0.8$ \\
\hline BMMSCs donor 4 & $86.15 \pm 0.65$ & $98.8 \pm 0.85$ & $99.9 \pm 0.1$ & $0.15 \pm 0.05$ & $1.75 \pm 0.65$ \\
\hline SFMSCs donor 4 & $95.3 \pm 0.1$ & $98.8 \pm 0.8$ & $99.4 \pm 0.4$ & $1.72 \pm 0.02$ & $6.15 \pm 1.4$ \\
\hline BMMSCs donor 5 & $99.9 \pm 0.05$ & $99.6 \pm 0.1$ & $99.8 \pm 0$ & $3.5 \pm 0.3$ & $4.1 \pm 0.9$ \\
\hline SFMSCs donor 5 & $96.4 \pm 2.8$ & $99.4 \pm 0.1$ & $99.7 \pm 0.05$ & $0.99 \pm 0.5$ & $21 \pm 6$ \\
\hline
\end{tabular}
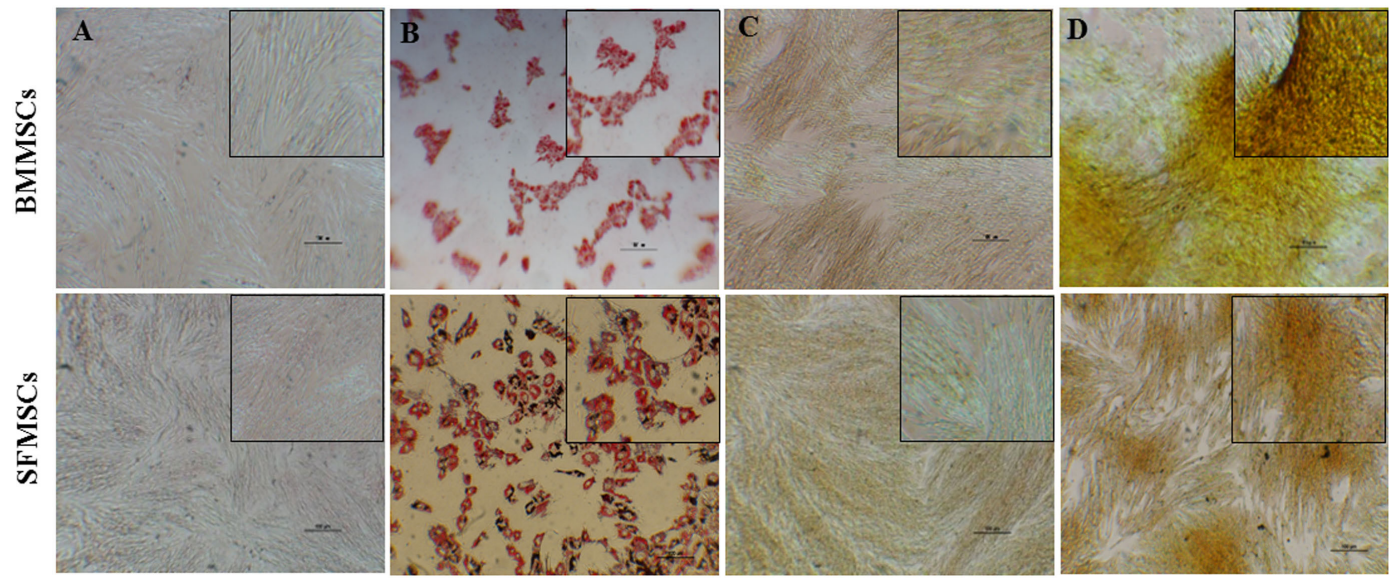

FIGURE 3 | Representative images of adipogenic and osteogenic differentiation assays. Bone marrow-derived mesenchymal stem cells (BMMSCs) and synovial fluid-derived mesenchymal stem cells (SFMSCs) were induced to differentiate using lineage-specific media. (A,B) Oil Red O staining of cells cultured for 7 days in undifferentiated and adipogenic differentiation medium, respectively. (C,D) Alizarin red staining of cells cultured for 14 days in undifferentiated and osteogenic differentiation medium, respectively. Scale bar $=100 \mu \mathrm{m}$

\section{Chondrogenesis}

Chondrogenic differentiation was demonstrated by the deposition of GAG by Alcian Blue staining. Striking differences were observed, these differences in staining were supported by the variations in the GAG content in each of the differentiated sample (Figure 4).

The GAG content was measured and compared between the BMMSCs and SFMSCs within each donor. SFMSCs of three donors exhibited a significant increase in GAG content compared to the BMMSCs $(P<0.0001-0.006)$. Other two donors, the pattern was reversed and the BMMSCs exhibited a significant increase $(P<0.0001-0.003)$ in GAG content compared to the SFMSCs (Figure 4).

The variations in chondrogenesis were further evaluated by assessing the expression of chondrogenic protein markers during differentiation. IF demonstrated the expression of SOX9, Aggrecan, and Col II at 3, 14, and 14 days, respectively, in both cell types (Figures $\mathbf{5}$ and $\mathbf{6}$ ). However, quantitative analyses showed significant variation in their expression levels. The expression levels of all three target proteins were significantly upregulated in SFMSCs compared to BMMSCs from at least two donors. Aggrecan was the only protein marker that was significantly upregulated in BMMSCs cultured from two donors.

\section{DISCUSSION}

Effective cartilage tissue engineering using MSCs mostly depends on the capacity of cells to proliferate, differentiate, and establish cartilage in vivo. $\mathrm{BM}$ and $\mathrm{AD}$ have been the most extensively studied and used sources for obtaining MSCs. To alleviate some of the technical challenges associated with the tissue harvest and properties of MSCs cultured ex vivo from BM and AD, recently, $\mathrm{SF}$ and the synovium membrane have been identified as alternative sources.

Reports have demonstrated that SFMSCs are multipotent and exhibit properties of adult MSCs; in addition, SF collection is relatively easy and less invasive $(17,18,26,29)$. Studies have reported the isolation, identification, and biological properties of equine SFMSCs and BMMSCs $(17,18,23,30)$. However, a detailed pairwise comparison with respect to the growth, 


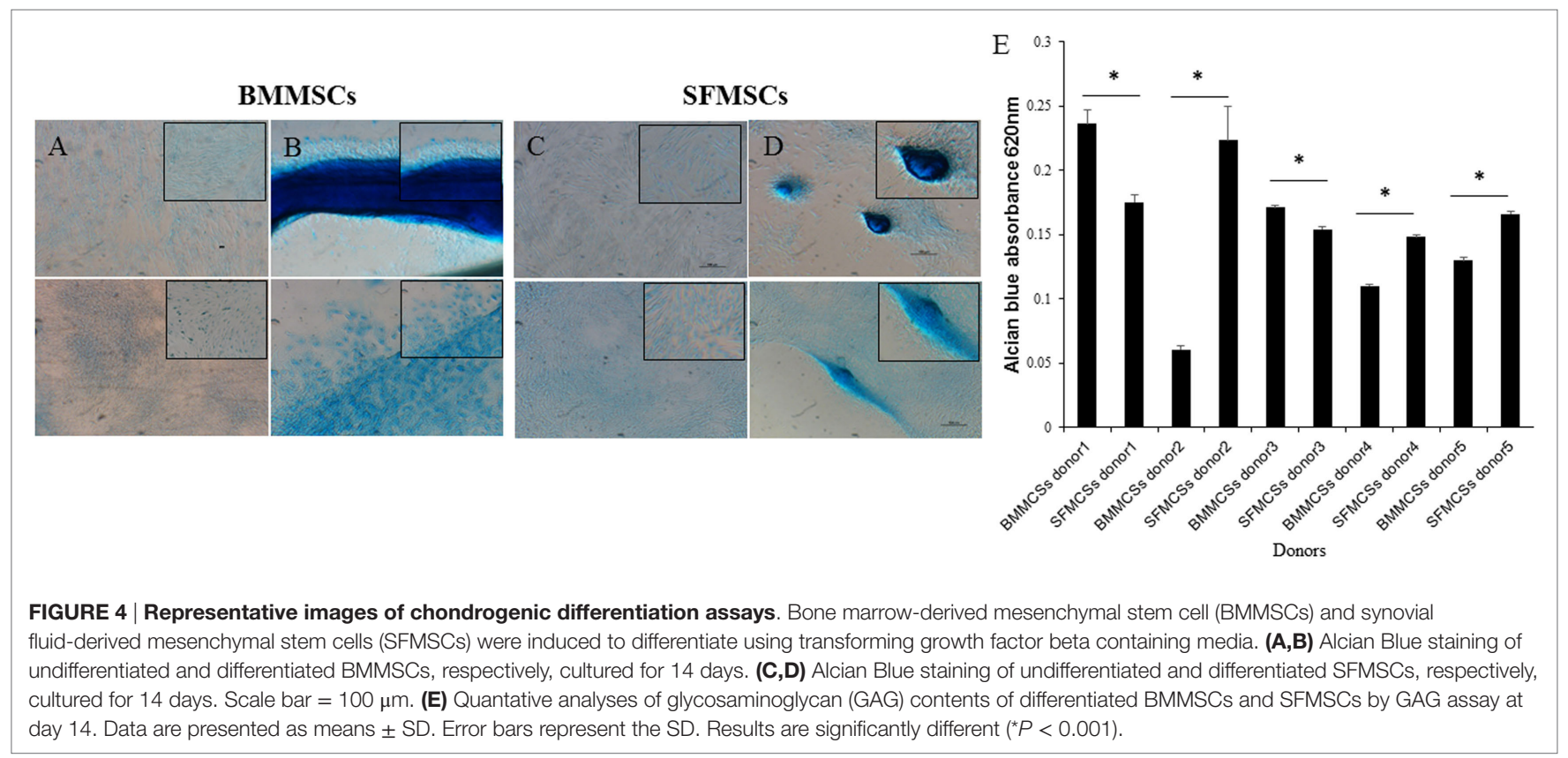

A

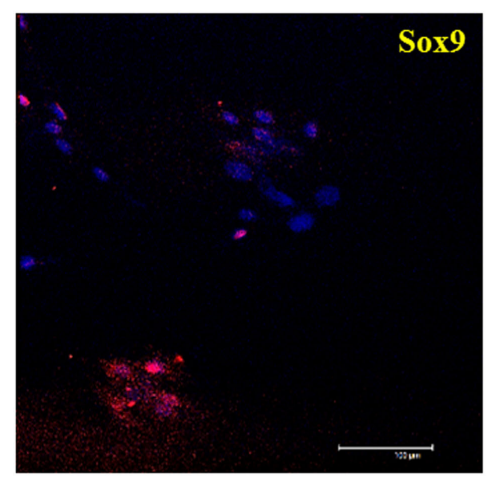

SFMSCs

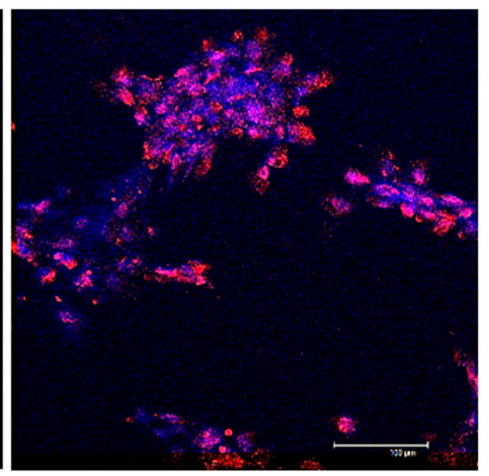

B

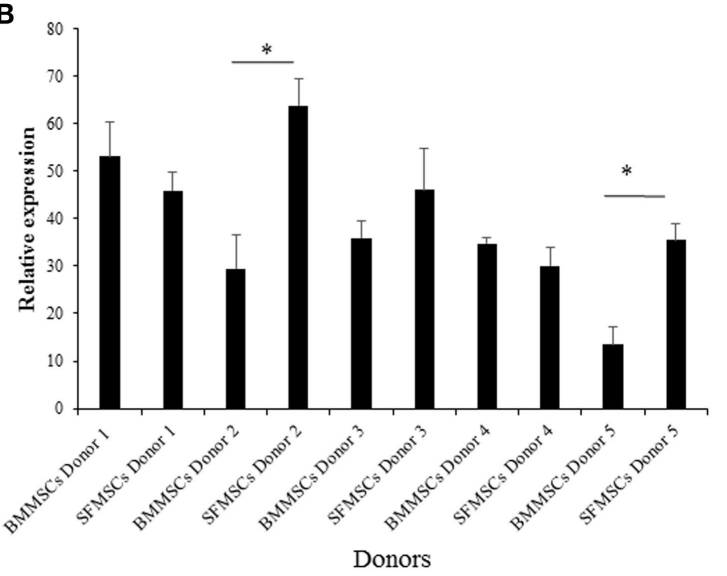

FIGURE 5 | Representative images to show the expression of cartilage-specific protein marker (SRY-type HMG box9; SOX9) detected by immunofluorescence in cultured bone marrow-derived mesenchymal stem cell (BMMSCs) and synovial fluid-derived mesenchymal stem cell (SFMSCs) from one donor. (A) Cells were stained for SOX9 (red) and DAPI (blue). (B) Quantitation of SOX9 expression in BMMSCs and SFMSCs. Results are significantly different $\left({ }^{\star} P<0.004\right)$. Scale bar $=100 \mu \mathrm{m}$.

chondrogenic differentiation, and expression of chondrogenic proteins between BMMSCs and SFMSCs from the same donor has not been reported. The impact of donor is particularly critical for autologous treatment regimens and in deciding whether such a cell-based therapy represents the appropriate treatment option for an individual patient. Thus, the purpose of this study was to investigate in vitro properties of SFMSCs and BMMSCs generated from five donors with a focus on their cartilage forming potential.

In this study, MSCs adhered to tissue culture flasks and proliferated showing spindle, fibroblast-like morphology that is typical of MSCs. SF harvested from a joint has limited number of progenitor cells and hence requires in vitro expansion to generate sufficient numbers of viable MSCs required for the treatment of an injury $(18,31)$. A linear increase in both BMMSCs and SFMSCs from five equine donors over a period of 8 days (Figure 2), suggested that in presence of an optimal growth medium containing $10 \%$ fetal bovine serum, both BMMSCs and SFMSCs are viable and can generate sufficient numbers of MSCs for clinical application (27). Similar expression levels of the three commonly reported CD markers, CD29, CD44, and CD90, in BMMSCs and SFMSCs (Table 3) from all donors further confirmed the "stemness" of these primary cultures. Most importantly, the high level of 


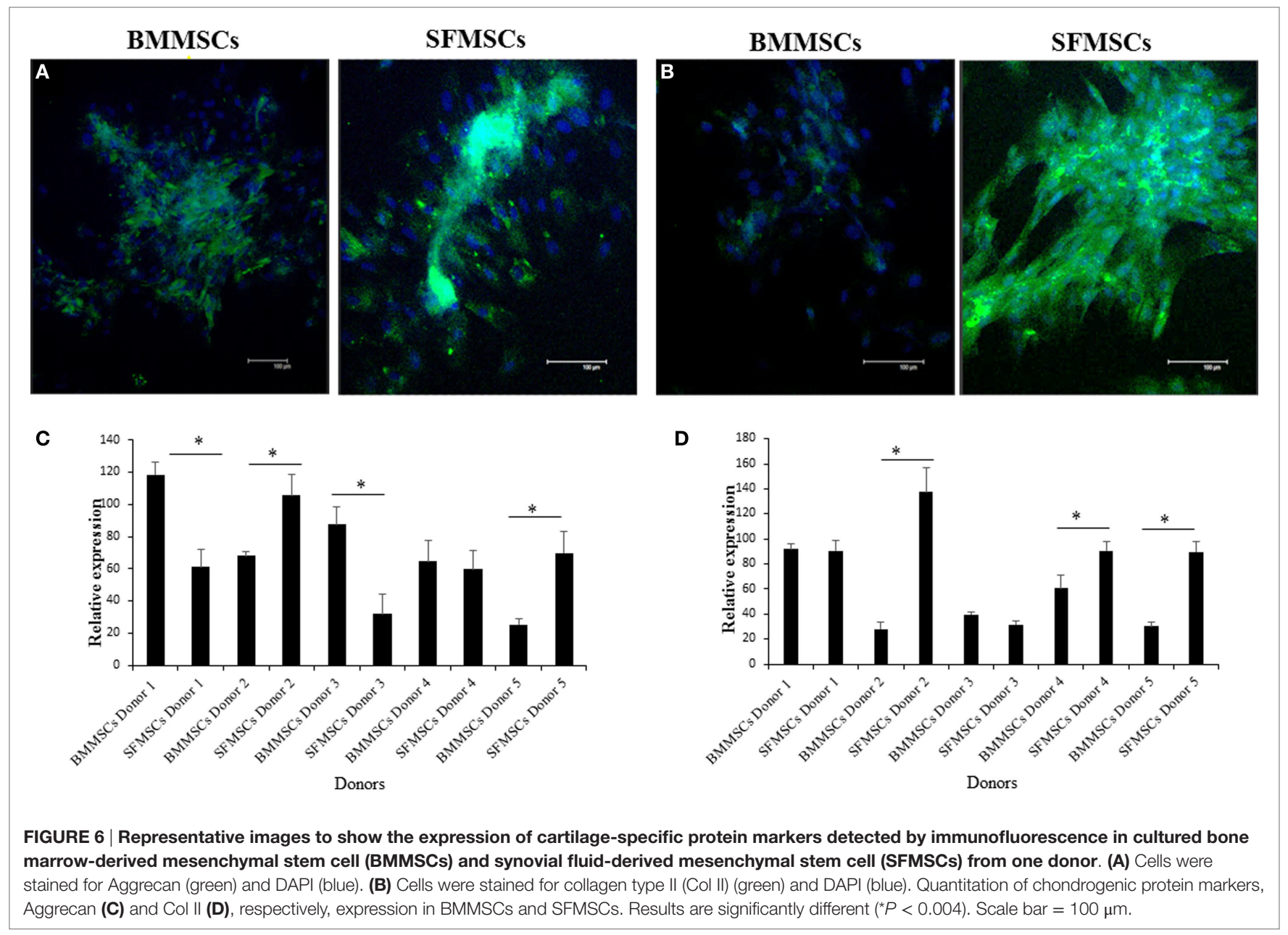

MHC-II expression suggests that in contrast to the BMMSCs, the SFMSCs may not be used as an allogeneic source of MSCs for therapy; however, in vivo investigation is needed.

The potential for trilineage differentiation is yet another important biological property that should always be used to verify the multipotentiality of MSCs. Differences have been reported in these assays in MSCs derived from various sources (14, 19, 30, 32). Consistent with the previous studies, cells from both sources differentiated into adipocytes, osteocytes, and chondrocytes within 7,14 , and 14 days, respectively $(14,18,32)$. Of relevance to this study, even though both BMMSCs and SFMSCs from all donors showed the potency to differentiate into chondrogenic lineage, the process exhibited varying degrees. Subjectively, the Alcian Blue staining and quantitatively measured by GAG content, there was a significant upregulation in the chondrogenic potentials of SFMSCs from three out of five donors (Figure 4). On the other hand, the chondrogenic potential of BMMSCs from two donors were upregulated relative to SFMSCs. Thus, results suggest that all SFMSCs are not necessarily superior to BMMSCs in their chondrogenic potential but demonstrate donor-to-donor variation.

Mesenchymal cell differentiation into chondrocytes is a complex process that involves a very well-orchestrated signaling pathway of growth factors (33). In our study, we investigated the changes in expression of specific key markers that coordinate this process in the hope of understanding what makes one MSC culture better suited for chondrogenesis compared to the other. It has been shown that SOX9, a master regulatory factor, regulates a specific set of genes in chondrocytes $(34,35)$. Aggrecan and Col II are the major components in the articular cartilage, which play an important role in chondrocytes differentiation and chondrocyte-matrix interactions $(36,37)$. Col II is an important marker for hyaline cartilage, which is valuable when cartilage healing is evaluated in vivo. Our results indicate that both BMMSCs and SFMSCs could express SOX9, Aggrecan, and Col II in all primary cultures, however, with varying levels.

The significant upregulation of SOX9 in SFMSCs cultured from two donors, along with a significantly higher GAG content and Aggrecan, suggests that the increased levels of these regulatory proteins may be responsible for their higher chondrogenic potential. Additionally, the significant upregulation of Col II in SFMSCs from three donors suggests that the potential for regeneration of hyaline cartilage is higher in these SFMSCs (Figure 6). Conversely, BMMSCs from other two donors demonstrate significant increase in GAG content coupled with the expression of Aggrecan, but there is no change in the SOX9 expression 
(Figure 5), suggesting that the chondrogenic potentials in these two donors may be influenced by some other factors unknown at this time. These results suggest that since chondrogenesis is a complex process, it can be affected at multiple points by the variation in expression of chondrocyte progenitor proteins thus leading to significant changes in the process itself. As a result, all SFMSCs need not be superior to BMMSCs but are dependent on the donor.

Another highlight of this study is that we were able to detect the expression of key chondrocyte proteins in equine MSCs, which can prove to be very helpful in future in vitro and in vivo experiments. In equine medicine, we lack equine-specific antibodies to detect the expression of SOX9, Aggrecan, and Col II limiting us to use those that are raised against mouse or human target proteins (38).

In conclusion, the use of MSCs for cell and tissue regeneration depends on the capability to in vitro expand and at the same time maintain their multipotency. Large differences among the donors in growth and response to chondrogenic signals can limit their application. Careful evaluation of various samples from the same donor may improve the clinical outcomes and progress tissue engineering applications. In this study, we did prove our hypothesis and were able to accomplish a pairwise comparison

\section{REFERENCES}

1. Ishiguro N, Kojima T, Poole AR. Mechanism of cartilage destruction in osteoarthritis. Nagoya J Med Sci (2002) 65:73-84.

2. McIlwraith CW, Frisbie DD, Kawcak CE. The horse as a model of naturally occurring osteoarthritis. Bone Joint Res (2012) 1:297-309. doi:10.1302/20463758.111.2000132

3. Wilsher S, Allen WR, Wood JL. Factors associated with failure of thoroughbred horses to train and race. Equine Vet J (2006) 38:113-8. doi:10.2746/ 042516406776563305

4. Bhatia D, Bejarano T, Novo M. Current interventions in the management of knee osteoarthritis. JPharm Bioallied Sci (2013) 5:30-8. doi:10.4103/ 0975-7406.106561

5. Goodrich LR, Nixon AJ. Medical treatment of osteoarthritis in the horse - a review. Vet J (2006) 171:51-69. doi:10.1016/j.tvjl.2004.07.008

6. Frisbie DD, Bowman SM, Colhoun HA, DiCarlo EF, Kawcak CE, McIlwraith CW. Evaluation of autologous chondrocyte transplantation via a collagen membrane in equine articular defects: results at 12 and 18 months. Osteoarthritis Cartilage (2008) 16:667-79. doi:10.1016/j.joca.2007.09.013

7. Harris JD, Siston RA, Pan X, Flanigan DC. Autologous chondrocyte implantation: a systematic review. J Bone Joint Surg Am (2010) 92:2220-33. doi:10.2106/JBJS.J.00049

8. Fortier LA, Travis AJ. Stem cells in veterinary medicine. Stem Cell Res Ther (2011) 2:9-9. doi:10.1186/scrt50

9. Frisbie DD, Smith RK. Clinical update on the use of mesenchymal stem cells in equine orthopaedics. Equine Vet J (2010) 42:86-9. doi:10.2746/0425164 09X477263

10. Taylor SE, Smith RK, Clegg PD. Mesenchymal stem cell therapy in equine musculoskeletal disease: scientific fact or clinical fiction? Equine Vet J (2007) 39:172-80. doi:10.2746/042516407X180868

11. Law S, Chaudhuri S. Mesenchymal stem cell and regenerative medicine: regeneration versus immunomodulatory challenges. Am J Stem Cells (2013) 2:22-38.

12. Mobasheri A, Kalamegam G, Musumeci G, Batt ME. Chondrocyte and mesenchymal stem cell-based therapies for cartilage repair in osteoarthritis and related orthopaedic conditions. Maturitas (2014) 78:188-98. doi:10.1016/ j.maturitas.2014.04.017 of SFMSCs and BMMSCs in five equine donors. Using in vitro molecular and cellular assays, we did identify donors with superior and inferior SFMSCs, which can greatly affect their clinical applications. Further in vivo experiments using controlled animal models are necessary to address the performance of SFMSCs, prior to their use in clinical cases.

\section{AUTHOR CONTRIBUTIONS}

$\mathrm{MZ}, \mathrm{CC}$, and MD contributed to study design, data collection, and study execution. NM assisted in data analysis and interpretation; all the authors were involved in the preparation of the manuscript.

\section{ACKNOWLEDGMENTS}

The authors thank John Dunlap, image center, University of Tennessee, for his helping in IF images and Xiaocun Sun, office of information technology, University of Tennessee, for her helping in data analysis. This work was financed by Egyptian Cultural and Educational Bureau (ECEB), and University of Tennessee, Center of Excellence in Livestock Diseases and Human Health (Grant number R181710145).

13. Arnhold SJ, Goletz I, Klein H, Stumpf G, Beluche LA, Rohde C, et al. Isolation and characterization of bone marrow-derived equine mesenchymal stem cells. Am J Vet Res (2007) 68:1095-105. doi:10.2460/ajvr.68.10.1095

14. Barberini DJ, Freitas NP, Magnoni MS, Maia L, Listoni AJ, Heckler MC, et al. Equine mesenchymal stem cells from bone marrow, adipose tissue and umbilical cord: immunophenotypic characterization and differentiation potential. Stem Cell Res Ther (2014) 5:25. doi:10.1186/scrt414

15. Braun J, Hack A, Weis-Klemm M, Conrad S, Treml S, Kohler K, et al. Evaluation of the osteogenic and chondrogenic differentiation capacities of equine adipose tissue-derived mesenchymal stem cells. Am J Vet Res (2010) 71:1228-36. doi:10.2460/ajvr.71.10.1228

16. Dhar M, Neilsen N, Beatty K, Eaker S, Adair H, Geiser D. Equine peripheral blood-derived mesenchymal stem cells: isolation, identification, trilineage differentiation and effect of hyperbaric oxygen treatment. Equine Vet J (2012) 44:600-5. doi:10.1111/j.2042-3306.2011.00536.x

17. Murata D, Miyakoshi D, Hatazoe T, Miura N, Tokunaga S, Fujiki M, et al. Multipotency of equine mesenchymal stem cells derived from synovial fluid. Vet J (2014) 202:53-61. doi:10.1016/j.tvjl.2014.07.029

18. Prado AA, Favaron PO, da Silva LC, Baccarin RY, Miglino MA, Maria DA. Characterization of mesenchymal stem cells derived from the equine synovial fluid and membrane. BMC Vet Res (2015) 11:281. doi:10.1186/ s12917-015-0531-5

19. Vidal MA, Robinson SO, Lopez MJ, Paulsen DB, Borkhsenious O, Johnson JR, et al. Comparison of chondrogenic potential in equine mesenchymal stromal cells derived from adipose tissue and bone marrow. Vet Surg (2008) 37:713-24. doi:10.1111/j.1532-950X.2008.00462.x

20. Baksh D, Yao R, Tuan RS. Comparison of proliferative and multilineage differentiation potential of human mesenchymal stem cells derived from umbilical cord and bone marrow. Stem Cells (2007) 25:1384-92. doi:10.1634/ stemcells.2006-0709

21. Ketterl N, Brachtl G, Schuh C, Bieback K, Schallmoser K, Reinisch A, et al. A robust potency assay highlights significant donor variation of human mesenchymal stem/progenitor cell immune modulatory capacity and extended radio-resistance. Stem Cell Res Ther (2015) 6:236. doi:10.1186/s13287-0150233-8

22. Carter-Arnold JL, Neilsen NL, Amelse LL, Odoi A, Dhar MS. In vitro analysis of equine, bone marrow-derived mesenchymal stem cells demonstrates 
differences within age- and gender-matched horses. Equine Vet J (2014) 46:589-95. doi:10.1111/evj.12142

23. Fortier LA, Nixon AJ, Williams J, Cable CS. Isolation and chondrocytic differentiation of equine bone marrow-derived mesenchymal stem cells. Am $J$ Vet Res (1998) 59:1182-7.

24. Orth P, Rey-Rico A, Venkatesan JK, Madry H, Cucchiarini M. Current perspectives in stem cell research for knee cartilage repair. Stem Cells Cloning (2014) 7:1-17. doi:10.2147/SCCAA.S42880

25. McCarthy HE, Bara JJ, Brakspear K, Singhrao SK, Archer CW. The comparison of equine articular cartilage progenitor cells and bone marrow-derived stromal cells as potential cell sources for cartilage repair in the horse. Vet $J$ (2012) 192:345-51. doi:10.1016/j.tvjl.2011.08.036

26. Shirasawa S, Sekiya I, Sakaguchi Y, Yagishita K, Ichinose S, Muneta T. In vitro chondrogenesis of human synovium-derived mesenchymal stem cells: optimal condition and comparison with bone marrow-derived cells. JCell Biochem (2006) 97:84-97. doi:10.1002/jcb.20546

27. de Sousa EB, Casado PL, Moura Neto V, Duarte ME, Aguiar DP. Synovial fluid and synovial membrane mesenchymal stem cells: latest discoveries and therapeutic perspectives. Stem Cell Res Ther (2014) 5:112. doi:10.1186/ scrt501

28. Smith RK, Korda M, Blunn GW, Goodship AE. Isolation and implantation of autologous equine mesenchymal stem cells from bone marrow into the superficial digital flexor tendon as a potential novel treatment. Equine Vet $J$ (2003) 35:99-102. doi:10.2746/042516403775467388

29. Jones BA, Pei M. Synovium-derived stem cells: a tissue-specific stem cell for cartilage engineering and regeneration. Tissue Eng Part B Rev (2012) 18:301-11. doi:10.1089/ten.TEB.2012.0002

30. Ranera B, Ordovás L, Lyahyai J, Bernal ML, Fernandes F, Remacha AR, et al. Comparative study of equine bone marrow and adipose tissue-derived mesenchymal stromal cells. Equine Vet J (2012) 44:33-42. doi:10.1111/ j.2042-3306.2010.00353.x

31. Godwin EE, Young NJ, Dudhia J, Beamish IC, Smith RK. Implantation of bone marrow-derived mesenchymal stem cells demonstrates improved outcome in horses with overstrain injury of the superficial digital flexor tendon. Equine Vet J (2012) 44:25-32. doi:10.1111/j.2042-3306.2011.00363.x
32. Burk J, Ribitsch I, Gittel C, Juelke H, Kasper C, Staszyk C, et al. Growth and differentiation characteristics of equine mesenchymal stromal cells derived from different sources. Vet $J$ (2013) 195:98-106. doi:10.1016/j.tvjl. 2012.06.004

33. Bobick BE, Chen FH, Le AM, Tuan RS. Regulation of the chondrogenic phenotype in culture. Birth Defects Res C Embryo Today (2009) 87:351-71. doi:10.1002/bdrc.20167

34. Henry SP, Liang S, Akdemir KC, de Crombrugghe B. The postnatal role of Sox9 in cartilage. J Bone Miner Res (2012) 27:2511-25. doi:10.1002/ jbmr.1696

35. Tuli R, Tuli S, Nandi S, Huang X, Manner PA, Hozack WJ, et al. Transforming growth factor-beta-mediated chondrogenesis of human mesenchymal progenitor cells involves $\mathrm{N}$-cadherin and mitogen-activated protein kinase and Wnt signaling cross-talk. J Biol Chem (2003) 278:41227-36. doi:10.1074/jbc. M305312200

36. Kiani C, Chen L, Wu YJ, Yee AJ, Yang BB. Structure and function of aggrecan. Cell Res (2002) 12:19-32. doi:10.1038/sj.cr.7290106

37. Chen CW, Tsai YH, Deng WP, Shih SN, Fang CL, Burch JG, et al. Type I and II collagen regulation of chondrogenic differentiation by mesenchymal progenitor cells. J Orthop Res (2005) 23:446-53. doi:10.1016/j.orthres.2004.09.002

38. Ibrahim S, Steinbach F. Non-HLDA8 animal homologue section anti-leukocyte $\mathrm{mAbs}$ tested for reactivity with equine leukocytes. Vet Immunol Immunopathol (2007) 119:81-91. doi:10.1016/j.vetimm.2007.06.033

Conflict of Interest Statement: The authors declare that they have no competing interests. A research report of a part of this work was presented at the 2015 North American Veterinary Regenerative Medicine Association (NAVRMA), Monterey, CA, USA.

Copyright $\odot 2017$ Zayed, Caniglia, Misk and Dhar. This is an open-access article distributed under the terms of the Creative Commons Attribution License (CC BY). The use, distribution or reproduction in other forums is permitted, provided the original author(s) or licensor are credited and that the original publication in this journal is cited, in accordance with accepted academic practice. No use, distribution or reproduction is permitted which does not comply with these terms. 